\title{
Optimal LQG controller to adjust the rudder supplying water to the turbine of small and medium hydro power plants
}

\section{Controlador LQG óptimo para ajustar el timón que suministra agua a la turbina de pequeñas y medianas centrales hidroeléctricas}

\section{Controlador LQG ideal para ajuste do leme que fornece água para a turbina de pequenas e médias usinas hidrelétricas}

\author{
Trung Dang Tien ${ }^{1}$ \\ Giang Le Ngoc ${ }^{2}$ \\ Tinh Tran Xuan ${ }^{3}$ \\ Linh Nguyen Thi Dieu ${ }^{4}$
}

Received: March $15^{\text {th }}, 2021$

Accepted: June $20^{\text {th }}, 2021$

Available: September $6^{\text {th }}, 2021$

How to cite this article:

Trung Dang Tien, Giang Le Ngoc, Tinh Tran Xuan, Linh Nguyen Thi Dieu, "Optimal LQG controller to adjust the rudder supplying water to the turbine of small and medium hydro power plants," Revista Ingeniería Solidaria, vol. 17, no. 3, 2021. doi: https://doi.org/10.16925/2357-6014.2021.03.07

Research article. https://doi.org/10.16925/2357-6014.2021.03.07

1 Electric Power University in Vietnam

Email: dangtientrung@gmail.com

ORCID: https://orcid.org/0000-0001-7388-8037

2 AD-AF Academy of Viet Nam, Son Tay, Ha Noi, Viet Nam

Email: lengocgianglinh@gamail.com

ORCID: https://orcid.org/0000-0002-0198-2857

3 AD-AF Academy of Viet Nam, Son Tay, Ha Noi, Viet Nam

Email: tinhpk79@gmail.com

ORCID: https://orcid.org/0000-0003-3451-7721

4 Faculty of Electronics Engineering Technology, Hanoi University of Industry

Email: dieulinh79@gmail.com

ORCID: https://orcid.org/ 0000-0001-8596-392X 
2 Optimal LQG controller to adjust the rudder supplying water to the turbine of small and medium hydro power plants

\section{Abstract}

Introduction: This paper is the result of the research "Optimal LQG controller to adjust the rudder supplying water to turbine of small and medium hydro power plants" developed at the Electric Power University in Vietnam in 2019 .

Problem: To maintain the frequency of the emitted voltage of the generator at the nominal value of $50 \mathrm{~Hz}$, the authors present a solution to apply optimal control theory to create a command to control the rudder angle to adjust the water flow into the turbine.

Objective: To present the order value formation algorithm used to stabilize the frequency of transmission voltage at $50 \mathrm{~Hz}$ standard value.

Methodology: In this paper, the laws to control the rudder supplying water to the turbine of small and medium hydro power plants are synthesized by optimal control theory. To establish optimal control rules, the paper proposes using the Kalman filter to estimate the state of the object. By that, the frequency of the generated voltage will be stabilized under changing load. The efficacy of the steady and dynamic performance of the control strategy was verified using Matlab/Simulink software.

Results: The proposed system can compensate for power fluctuations and is effective in terms of power regulation.

Conclusion: The algorithm presented in the paper is the basis for setting up the software when designing and manufacturing the turbine - generator combination. Applying this algorithm, the process of adjusting the transmitted power according to the required load will be performed with quality.

Originality: This paper's contribution lies in its employment of an effective optimal LQG control for varying operating conditions.

Limitations: The authors require more time to develop and test this algorithm prior to its implemention.

Keywords: Optimal control, rudder, hydropower turbine, stabilize the frequency.

\section{Resumen}




\section{Resumo}

\section{INTRODUCTION}

Article [1] presented a mathematical model describing the relationship between the rotation angle of the rudder to supply potential energy and kinetic energy of a water column for turbines of the combination "turbine + generator" in small and medium-sized hydroelectric power plants. However, the order value formation algorithm, to stabilize the frequency of transmission voltage at $50 \mathrm{~Hz}$ standard value, was not presented. In this paper, the authors present a solution to apply the optimal control theory to create a command to control the rudder angle to adjust the water flow into 
Optimal LQG controller to adjust the rudder supplying water to the turbine of small and medium hydro power plants

the turbine, thus maintaining the frequency of the emitted voltage of the generator at the nominal value of $50 \mathrm{~Hz}$, under changing load conditions and water column heights.

Proportional-integral structured optimal controllers are designed using a fullstate feedback control strategy employing performance index minimization criterion. Some traditional single/multiarea and restructured multiarea power system models from the literature are explored deliberately in the present study. The dynamic performance of optimal controllers is seen to be superior in comparison to integral/proportional-integral controllers tuned using some recently published modern heuristic optimization techniques. It is observed that optimal controllers show better system results in terms of minimum value of settling time, peak overshoot/undershoot, various performance indices, and oscillations corresponding to change in area frequencies and tie-line powers along with maximum value of minimum damping ratio in comparison to other controllers.

This paper's contribution lies in its employment of an effective optimal LQG control for varying operating conditions. Optimal LQG control is one of the most successful control algorithms and is widely used in handling multivariables and constraints. The designed control discipline is based on a mathematic model of a controlled object with the prescribed limit to acquire the optimal performance index [8-10]. According to previous research on PID control, the conventional control algorithm gives good results at infinite steady state; the only difficulty occurs when the reference trajectory is fluctuating $[2,3,4]$. This paper builds on the ideas presented in [5-7]. The performance of a controller using optimal LQG control and PID will be analyzed and discussed in the simulation section.

\section{CONTENT AND RESEARCH METHODS}

\subsection{Building control algorithm}

Article [1] described the relationship between the control signal to rotate the rudder and the rotation frequency of the turbine as follows:

$$
\begin{gathered}
T \frac{d \omega}{d t}+\omega=K \alpha+z_{1} \\
T_{\alpha} \frac{d^{2} \alpha}{d t^{2}}+\alpha=K_{u} U+z_{2}
\end{gathered}
$$


Where: $\alpha$ is the rudder opening angle; $\omega$ is the rotation frequency of the turbine; Parameters $T, T_{\alpha}, K, K_{u}$ depend on the pressure and flow rate of the water column; Parameter $z_{2}$ depends on the pressure of the water column; Parameter $z_{1}$ depends on the pressure and flow rate of the water column, and load consumption. In that paper, we also presented an algorithm to identify these uncertainty parameters.

Because the difference between the generating frequency and the reference frequency $\omega_{0}=2 \pi f_{0}=2 \pi 50=100 \pi$ ( $\mathrm{rad} / \mathrm{sec}$ ) is the basic information for forming the control signal, the following should be set:

$$
x_{1}=\omega-\omega_{0}
$$

$$
\omega=x_{1}+\omega_{0}
$$

(4) can be substituted into (1) to get the equation:

$$
T \frac{d x_{1}}{d t}+x_{1}+\omega_{0}=K \alpha+z_{1}
$$

Set:

$$
\begin{gathered}
x_{2}=\alpha \\
x_{3}=\dot{\alpha}=\frac{d \alpha}{d t}=\dot{x}_{2}=\frac{d x_{2}}{d t}
\end{gathered}
$$

Equation (2) will have the following form:

$$
T_{\alpha} \dot{x}_{3}+x_{2}=K_{u} U+z_{2}
$$

From the three linear differential equations (5), (7), (8) has the following linear dynamic system:

$$
\dot{x}_{1}=-\frac{1}{T} x_{1}+\frac{K}{T} x_{2}+\frac{z_{1}-\omega_{0}}{T}
$$


6 Optimal LQG controller to adjust the rudder supplying water to the turbine of small and medium hydro power plants

$$
\begin{gathered}
\dot{x}_{2}=x_{3} \\
\dot{x}_{3}=-\frac{x_{2}}{T_{\alpha}}+\frac{K_{u}}{T_{\alpha}} U+\frac{z_{2}}{T_{\alpha}}
\end{gathered}
$$

Set state vector:

$$
X=\left(x_{1} x_{2} x_{3}\right)^{T}
$$

From the three equations (9), (10), (11) there are dynamic equations in the form of state space as follows:

$$
\dot{X}=A X+B U+C V
$$

Where:

$$
\begin{aligned}
& A=\left[\begin{array}{l}
a_{11} a_{12} a_{13} \\
a_{21} a_{22} a_{13} \\
a_{31} a_{32} a_{33}
\end{array}\right] ; B=\left[\begin{array}{l}
0 \\
0 \\
b
\end{array}\right] ; C=\left[\begin{array}{l}
c_{11} c_{12} \\
c_{21} c_{22} \\
c_{31} c_{32}
\end{array}\right] \\
& a_{11}=-\frac{1}{T} ; a_{12}=\frac{K}{T} ; a_{13}=0 \\
& a_{21}=0 ; a_{22}=0 ; a_{23}=1 \\
& a_{31}=0 ; a_{32}=-\frac{1}{T_{\alpha}} ; a_{33}=0 \\
& b=\frac{K_{u}}{T_{\alpha}} \\
& c_{11}=\frac{1}{T} ; c_{12}=0 ; c_{21}=0 ; c_{22}=0 ; c_{32}=0 ; c_{33}=\frac{1}{T_{\alpha}}
\end{aligned}
$$




$$
V=\left[\begin{array}{l}
v_{1} \\
v_{2}
\end{array}\right] ; v_{1}=z_{1}-\omega_{0} ; v_{2}=z_{2}
$$

The task of controlling generators in hydropower plants consists of two main sub tasks : control of generator excitation systems to stabilize the amplitude of the output voltage at the nominal value and control of the rudder supplying water to the turbine to stabilize the frequency of the output voltage at the nominal value.

The control of generator excitation systems has been published many times, so it is not considered in this paper. For all hydraulic generators currently available in Vietnam, the directional control algorithm often uses a PID control algorithm. However, this algorithm has different transient times when the load changes. In addition, the coefficient set for the PID controller is only reasonable when the parameters of matrices A, B, C in model (13) do not change.

During operation, due to the change of in power consumption load, the rotating frequency of the generator will change deviating from the standard frequency. $\left(\omega_{0}=100 \pi\right)$, if the load decreases then $\omega \geq \omega_{0}$, if the load increases then $\omega \leq \omega_{0}$. The control task must change the rudder angle so that the frequency returns to nominal frequency $\omega_{0}$, ie bringing the value of $x_{1}$ to zero $\left(x_{1} \rightarrow 0\right)$.

So we can set up the optimal control problem as follows:

Find the rule that changes the parameter value affecting the kinematic system (13) so that the function is:

$$
J=\frac{1}{2} \int_{0}^{T_{f}}\left(q x_{1}^{2}+r U^{2}\right) d t \rightarrow \min
$$

The function (21) can be written in the following standard form:

$$
J=\frac{1}{2} \int_{0}^{T_{f}}\left(X Q X^{T}+U R U^{T}\right) d t \rightarrow \min
$$

Where: $T_{f}$ is the end of the control process

$$
Q=\left[\begin{array}{l}
q_{11} q_{12} \\
q_{21} q_{22}
\end{array}\right] ; q_{11}=q ; q_{12}=q_{21}=q_{22}=0 ; R=[r]
$$

Apply the optimal control theory $[3,4]$ to solve the above problem to determine the law of value change $U$. First, set the Hamilton functions as follows: 
8 Optimal LQG controller to adjust the rudder supplying water to the turbine of small and medium hydro power plants

$$
H=\frac{1}{2}\langle X, Q X\rangle+\frac{1}{2}\langle U, R U\rangle+\langle A X, P\rangle+\langle B U, P\rangle+\langle C Z, P\rangle
$$

Notation $\langle$,$\rangle is the scalar product of two vectors. Vector P(t)$ is determined by:

$$
\dot{P}(t)=\frac{d p}{d t}=-\frac{\partial H}{\partial X}=-Q X(t)-A^{T} P(t)
$$

With boundary conditions:

$$
P\left(T_{f}\right)=0
$$

In the optimal orbit, satisfy the following equation:

$$
\frac{\partial H}{\partial U(t)}=0
$$

From (24) and (27) that:

$$
\frac{\partial H}{\partial U}=R U(t)+B^{t} P(t)=0
$$

So infer:

$$
U(t)=-R^{-1} B^{T} P(t)
$$

Vector $P(t)$ can be set as follows:

$$
P(t)=K_{x}(t) X(t)+K_{1}(t)
$$

To ensure the boundary condition (26), there are two conditions:

$$
\begin{gathered}
K_{x}\left(T_{f}\right)=0 \\
K_{1}\left(T_{f}\right)=0
\end{gathered}
$$


In this case, it is necessary to develop equations to determine the matrix $K_{x}(t)$ and the vector $K_{1}(t)$. From (30) we have:

$$
\dot{P}(t)=\dot{K}_{x}(t) X(t)+K_{x} \dot{X}(t)+\dot{K}_{1}(t)
$$

From (25) and (33) we have the following equation:

$$
\dot{K}_{x}(t) X(t)+K_{x} \dot{X}(t)+\dot{K}_{1}(t)=-Q X(t)-A^{T} P(t)
$$

From (13) and (34) we have the following equation:

$$
\dot{K}_{x}(t) X(t)+K_{x}(A X+B U+C V)+\dot{K}_{1}(t)=-Q X(t)-A^{T} P(t)
$$

Equivalent:

$$
\dot{K}_{x}(t) X(t)+K_{x}(A X+B U+C V)+\dot{K}_{1}(t)+Q X(t)+A^{T} P(t)=0
$$

From (29) and (36) we have the following equation:

$$
\dot{K}_{x}(t) X(t)+K_{x}\left(A X-B R^{-1} B^{T} P(t)+C V\right)+\dot{K}_{1}(t)+Q X(t)+A^{T} P(t)=0
$$

From (30) and (37) we have the following equation:

$$
\begin{aligned}
& \dot{K}_{x}(t) X(t)+K_{x}\left[A X-B R^{-1} B^{T}\left(K_{x}(t) X(t)+K_{1}(t)\right)+C V\right]+ \\
& +\dot{K}_{1}(t)+Q X(t)+A^{T}\left(K_{x}(t) X(t)+K_{1}(t)\right)=0
\end{aligned}
$$

Equivalent to:

$$
\begin{aligned}
& {\left[\dot{K}_{x}(t)+K_{x}(t) A+A^{T} K_{x}(t)-K_{x}(t) B R^{-1} B^{T} K_{x}(t)+Q\right] X(t)} \\
& +\left[\dot{K}_{1}(t)-\left(K_{x} B R^{-1} B^{T} K_{x}-A^{T}\right) K_{1}+K_{x} C V\right]=0
\end{aligned}
$$

For equation (39) to be true to all values of $X(t)$, then $K_{x}(t)$ and $K_{1}(t)$ must satisfy: 


$$
\dot{K}_{x}(t)+K_{x}(t) A+A^{T} K_{x}(t)-K_{x}(t) B R^{-1} B^{T} K_{x}(t)+Q=0
$$

$$
\dot{K}_{1}(t)-\left(K_{x} B R^{-1} B^{T} K_{x}-A^{T}\right) K_{1}+K_{x} C V=0
$$

Combining equation (40) with boundary conditions (31) and combining equation (41) with boundary conditions (32) provides a system of two differential equations to determine the matrix $K_{x}(t)$ and determine the vector $K_{1}(t)$ :

$$
\begin{gathered}
\dot{K}_{x}(t)=-K_{x}(t) A-A^{T} K_{x}(t)+K_{x}(t) B R^{-1} B^{T} K_{x}(t)-Q ; K_{x}\left(T_{f}\right)=0 \\
\left(K_{x}(t) B R^{-1} B^{T} K_{x}(t)-A^{T}\right) K_{1}-K_{x}(t) C V=\dot{K}_{1}(t) ; K_{1}\left(T_{f}\right)=0
\end{gathered}
$$

(42) shows that to determine $K_{x}(t)$, the matrices $A, B, R, Q$ must be known; this is the Riccati equation. From the boundary conditions of differential equation (43), to determine $K_{1}(t)$ at the present time t, one needs to have information about $\mathrm{V}$ in the future period $T_{f}$. Because the system of differential equations (43) is linear with the right boundary conditions, the solution of the equation is:

$$
K_{1}(t)=\int_{t}^{T_{f}} e^{\tilde{A} \tau} C V(\tau) d \tau
$$

Where:

$$
\tilde{A}=-\left(K_{x} B R^{-1} B^{T} K_{x}-A^{T}\right)
$$

According to [2], in case the integral time $T_{f}$ is long and the vector $V(t)$ does not change, the solution of equations (42) and (43) can be determined on the basis of solving the following algebraic equation system:

$$
-K_{x} A-A^{T} K_{x}+K_{x} B R^{-1} B^{T} K_{x}-Q=0
$$




$$
-\left(K_{x} B R^{-1} B^{T} K_{x}-A^{T}\right) K_{1}+K_{x} C V=0
$$

[2] presents the solution of the Riccati quadratic nonlinear equation system (46). After determining the coefficient matrix $K_{x}$, the solution of (47) is:

$$
K_{1}=\tilde{A}^{-1} K_{x} C V
$$

After determining $K_{x}$ and $K_{1}$, (29) and (30) provide the optimum control command to adjust the angle of the rudder supplying water to the turbine to rotate the generator as follows:

$$
U(t)=-R^{-1} B^{T} P(t)=-R^{-1} B^{T} K_{x} X-R^{-1} B^{T} K_{1}
$$

(49) shows that to synthesize an optimal control law, one needs to determine the matrix $K_{x}$ through solving algebraic equations (46). To determine the vector $\mathrm{Kt}$ according to (48) it is necessary to determine the state vector $X$ of the linear dynamic system (13), i.e. to measure or observe the deviation between the transmitter voltage frequency and the grid voltage frequency; the angle of the rudder and its opening speed. Thus, when designing and manufacturing the turbine and generator complex, it is necessary to arrange and install the corresponding measuring devices to measure or observe the information on the state of the dynamic system. In cases where it cannot be measured directly but must be observed, it is necessary to have the same algorithm and software to observe those parameters.

\subsection{The Kalman state observer $[11,12]$}

To establish optimal control rules of the form (13) one must have status information $X=\left(x_{1} x_{2} x_{3}\right)^{T}$, including:

- The difference between the actual frequency of the turbine rotation speed and the reference speed, ie $x_{1}=\omega-\omega_{0}$. This information can be measured by using a rotating-speed measuring device;

- The rudder angle $\alpha$, ie $x_{2}$. This information can be measured by the angle measuring device; 
- The rudder rotational speed $\dot{\alpha}$, ie $x_{3}$. This information is difficult to measure, so a Kalman filter must be applied.

Digitize two equations (10) and (11) with discrete steps $\Delta T$. There are two equations in the following differential form:

$$
\begin{aligned}
& x_{2}(k+1)=x_{2}(k)+x_{3}(k) \cdot \Delta T \\
& x_{3}(k+1)=x_{3}(k)+\left(-\frac{x_{2}(k)}{T_{\alpha}}+\frac{K_{u}}{T_{\alpha}} U(k)+\frac{z_{2}}{T_{\alpha}}\right) \cdot \Delta T+w(k)
\end{aligned}
$$

Where $w(k)$ is measurement error $U(k)$ and parameter identification error $z_{2}$. Using the Kalman filter algorithm:

$$
\begin{aligned}
& f_{1}\left(x_{2}, x_{3}\right)=x_{2}(k)+x_{3}(k) \cdot \Delta T \\
& f_{2}\left(x_{2}, x_{3}\right)=x_{3}(k)+\left(-\frac{x_{2}(k)}{T_{\alpha}}+\frac{K_{u}}{T_{\alpha}} U(k)+\frac{z_{2}}{T_{\alpha}}\right) \cdot \Delta T+w(k)
\end{aligned}
$$

With two components of state vector $X=\left(x_{1} x_{2}\right)^{T}, x_{2}$ is measurable, as is the rudder rotation angle $\alpha$ :

$$
z(k)=h(X(k))=x_{2}(k)+v(k)
$$

Where $v(t)$ is the measuring error of the rudder rotation angle due to the measuring device. The base transfer matrix of the Kalman filter is as follows:

$$
\Phi(k-1)=\frac{\partial F}{\partial X}=\left[\begin{array}{ll}
\frac{\partial f_{1}}{\partial x_{2}} & \frac{\partial f_{1}}{\partial x_{3}} \\
\frac{\partial f_{2}}{\partial x_{2}} & \frac{\partial f_{2}}{\partial x_{2}}
\end{array}\right]=\left[\begin{array}{cc}
1 & \Delta T \\
\frac{-\Delta T}{T_{\alpha}} & 1
\end{array}\right]
$$

From (52) the observation matrix of Kalman filter can be determined: 


$$
H(k)=\left(\frac{\partial h}{\partial X}\right)^{T}=\left[\begin{array}{ll}
\frac{\partial h}{\partial x_{2}} & \frac{\partial h}{\partial x_{3}}
\end{array}\right]=[10]
$$

From the system of equations (51), the noise intensity matrix is:

$$
G=\left[\begin{array}{l}
0 \\
1
\end{array}\right]
$$

The covariance matrix $Q, R$ of the Kalman filter procedure is:

$$
Q=\left[\sigma_{x}^{2}\right], R=\left[\sigma_{z}^{2}\right]
$$

Thus, it is possible to implement the Kalman filter algorithm to determine the composition of $x_{3}$, ie the rudder rotation angle, serving the synthesis of optimal control law. The filtering algorithm will include the following steps:

- $\quad$ Step 1: Establish initial matrix $P_{+}(k-1)$ as follows:

$$
P_{+}(k-1)=\left[\begin{array}{ll}
0 & 0 \\
0 & 0
\end{array}\right]
$$

- $\quad$ Step 2: Calculate the matrix $P_{-}(k)$ :

$$
P_{-}(k)=\Phi(k-1) P_{+}(k-1) \Phi^{T}(k-1)+G(k-1) Q(k-1) G^{T}(k-1)
$$

- $\quad$ Step 3: Calculate the Kalman coefficient matrix $K(k)$ :

$$
K(k)=P_{-}(k) H^{T}(k)\left(H(K) P_{-}(k) H^{T}(k)+R(k)\right)^{-1}
$$


Optimal LQG controller to adjust the rudder supplying water to the turbine of small and medium hydro power plants

- Step 4: Read the measured value of rudder angle:

- Step 5: Calculate vector:

$$
\hat{X}_{+}(k)=\hat{X}_{-}(k)+K(k)(Z(k)-\hat{Z}(k))
$$

- $\quad$ Step 6: Build state vector: $X=\left[x_{1} x_{2} x_{3}\right]^{T}$

Where: $x_{1}=\omega-\omega_{0}, x_{2}, x_{3}$ are the components of $\hat{X}_{+}(k)$ determined by (59).

- Step 7: Provide X to the control device to synthesize the optimal control law.

- $\quad$ Step 8: Calculate the matrix $P_{+}(k)$

$$
P_{+}(k)=(I-K(k) H(k)) P_{-}(k)
$$

- $\quad$ - Step 9: Calculate vector $\hat{X}_{-}(k)=\left(x_{2-}(k) x_{3-}(k)\right)^{T}$

$$
\begin{aligned}
& x_{2-}(k+1)=x_{2+}(k)+x_{3+}(k) \cdot \Delta T \\
& x_{3-}(k+1)=x_{3+}(k)+\left(-\frac{x_{2+}(k)}{T_{\alpha}}+\frac{K_{u}}{T_{\alpha}} U(k)+\frac{z_{2}}{T_{\alpha}}\right) \cdot \Delta T+w(t)
\end{aligned}
$$

The next filtering cycle will start from the second step (step 1 only performed once in the first cycle). The next filtering cycle is performed when the command $T=1$. The command $T=0$ instruction is issued by the control device when ordered to stop the operation of the turbine.

\section{RESULTS}

For all hydraulic generators currently available in Vietnam, the directional control algorithm often uses a PID control algorithm. Therefore, in this paper, the authors conducted simulations with 2 algorithms used for the directional control of hydraulic generators: PID control algorithm and the LQG control algorithm, to compare and evaluate control efficiency. 
The simulated object is a combination of turbine and generator with a counter-type turbine model (using both the potential and kinetic energy of the water column). The specifications of the unit are as follows:

- Rated power: $30 \mathrm{MW}$,

- The rotor has 4 pairs of poles, corresponding to the standard rotation frequency:

$$
\omega_{0}=\frac{2 \pi f_{0}}{4}=\frac{2 \pi 50}{4}=78,5(\operatorname{Rad} / \mathrm{s})
$$

- Moment of inertia of turbine and generator rotor: $J_{T}=160000 \mathrm{~kg} . \mathrm{m}^{2}$

- $\quad$ Smoothing coefficient $K_{c}=4,8$

- Rudder angle $\alpha$ change in scope: $0 \rightarrow 180^{\circ}$

- The height of the water column change from: $70 m \rightarrow 100 m$

- Coefficient $K_{y}$ change from: $5000 \rightarrow 20000$

- Coefficient $T_{y}$ change from: $5000 \rightarrow 20000$

- The inertia moment of rotating structure of rudder: $J=15.000 \mathrm{~kg} \cdot \mathrm{m}^{2}$

- Smoothing coefficient for rotating the rudders: $K_{\alpha}=0,5$

- Control coefficient of hydraulic rudder: $K_{x}=100$

- Hydrostatic torque: $M_{A}=150 \rightarrow 400$

- Control voltage $-24 \mathrm{~V} \rightarrow+24 \mathrm{~V}$

- Simulation parameters: $\mathrm{T}=0.02 ; \mathrm{Ta}=0.1 ; \mathrm{K}=10 ; \mathrm{Ku}=2$;

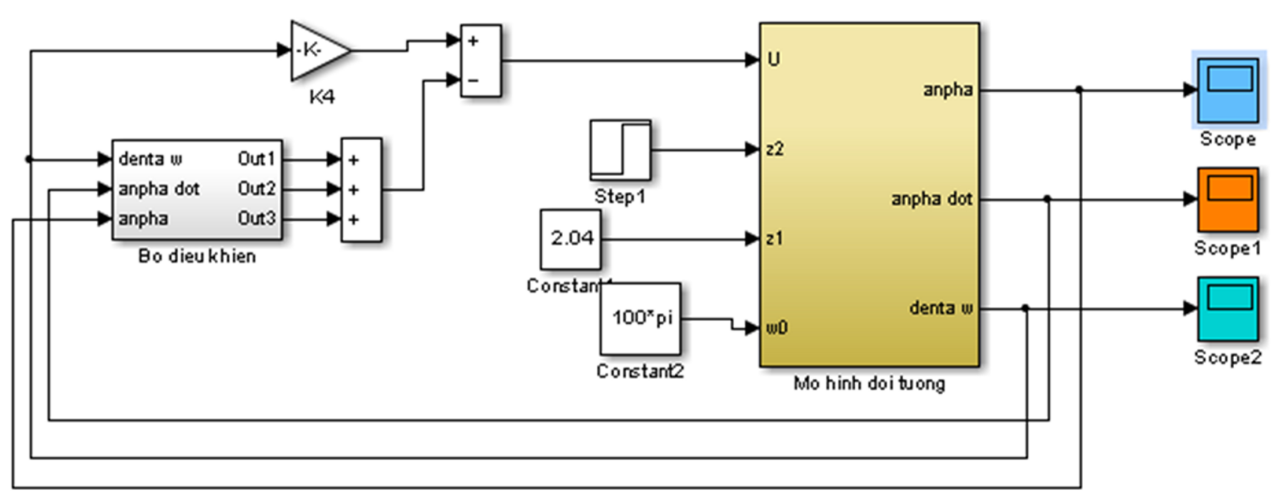

Figure 1. Simulation model of open algorithm for the rudder

Source: own work 
Optimal LQG controller to adjust the rudder supplying water to the turbine of small and medium hydro power plants

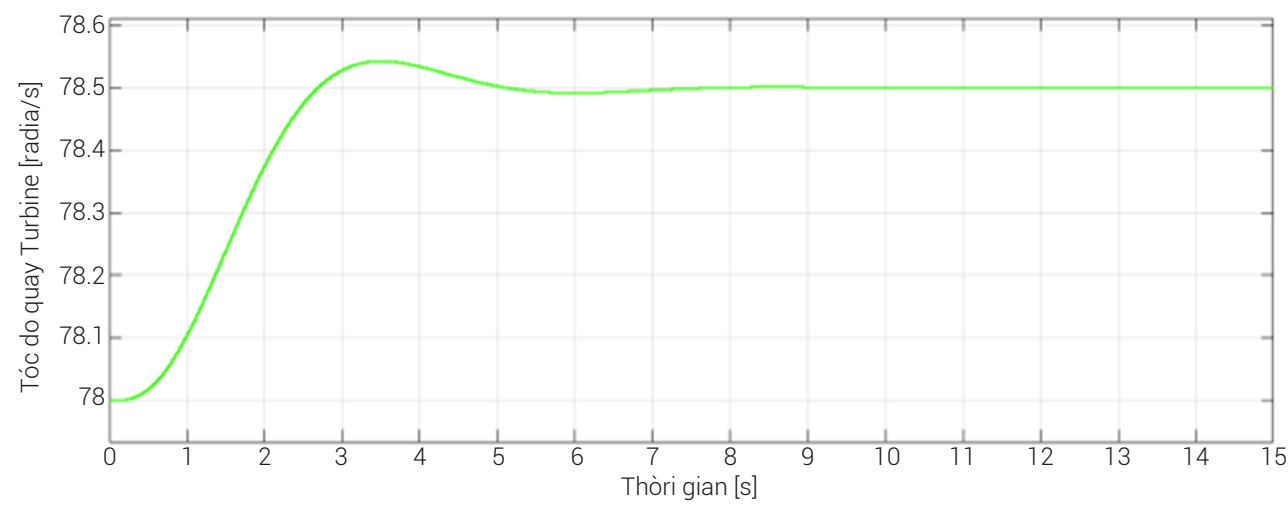

Figure 2. Turbine rotating frequency with optimal control law Source: own work

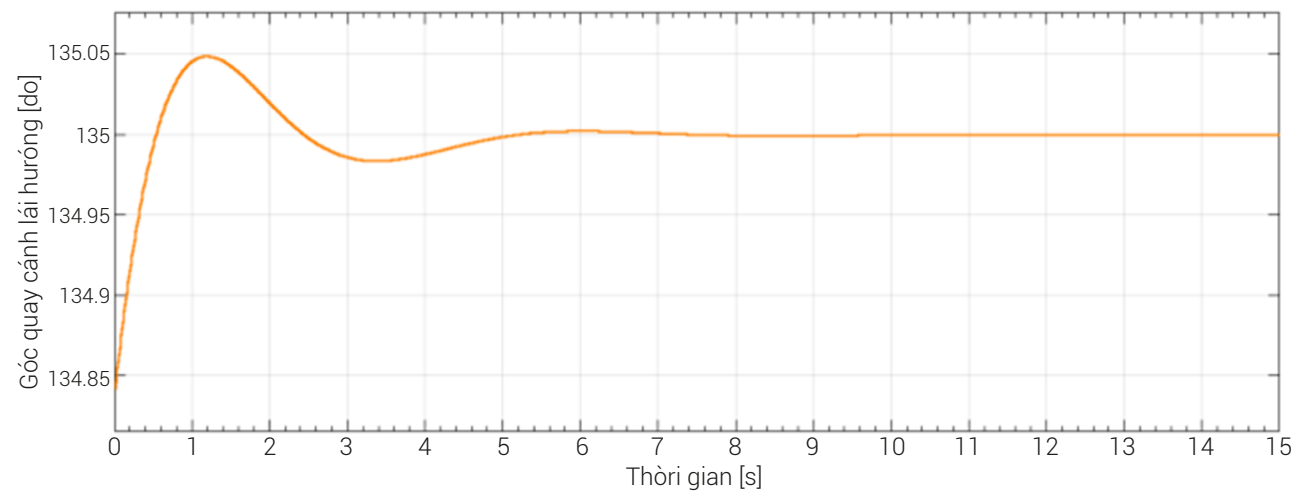

Figure 3. Rudder angle with optimal control law Source: own work

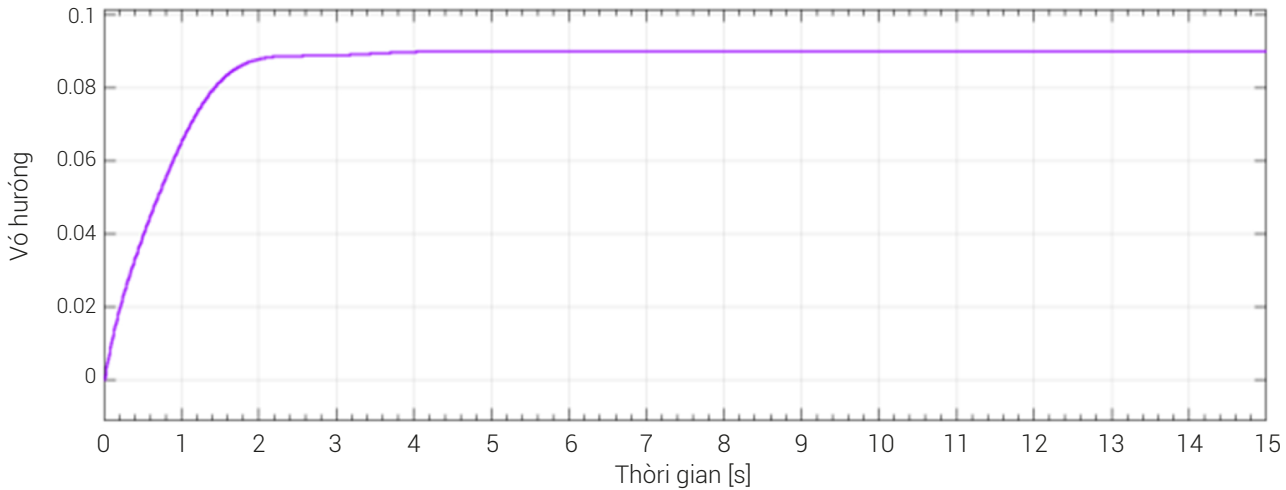

Figure 4. The objective function J with optimal control law Source: own work 


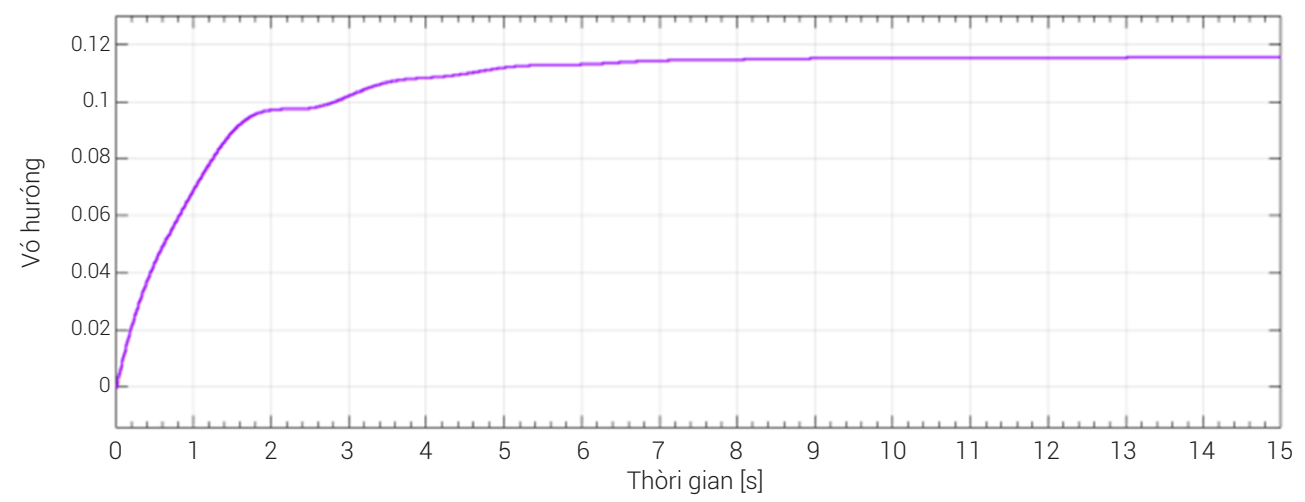

Figure 5. The objective function J with PID control law (Kx reduction) Source: own work

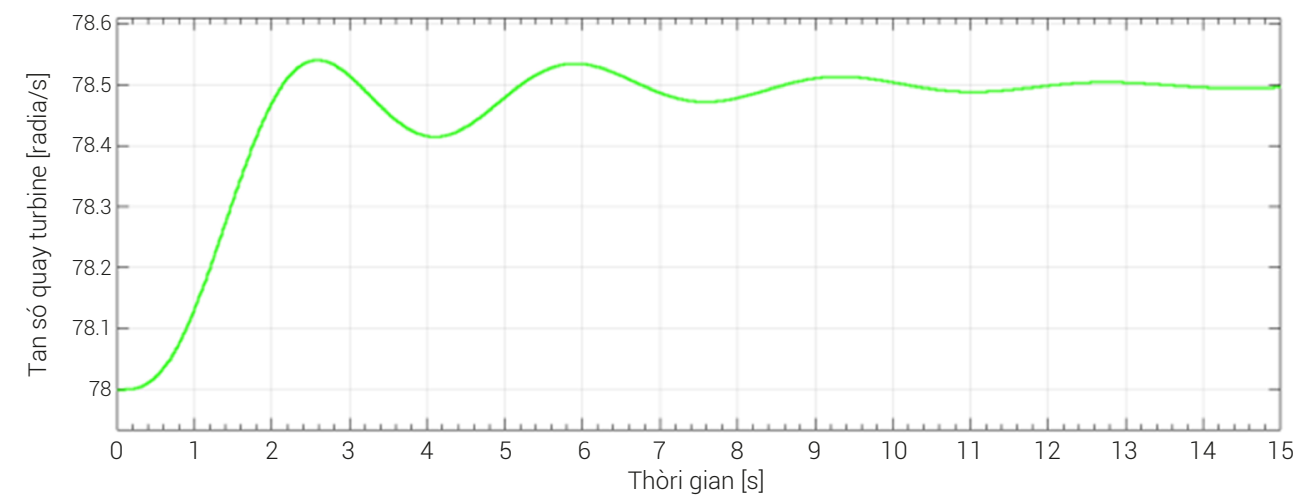

Figure 6. Turbine rotating frequency with PID control law (Kx reduction) Source: own work

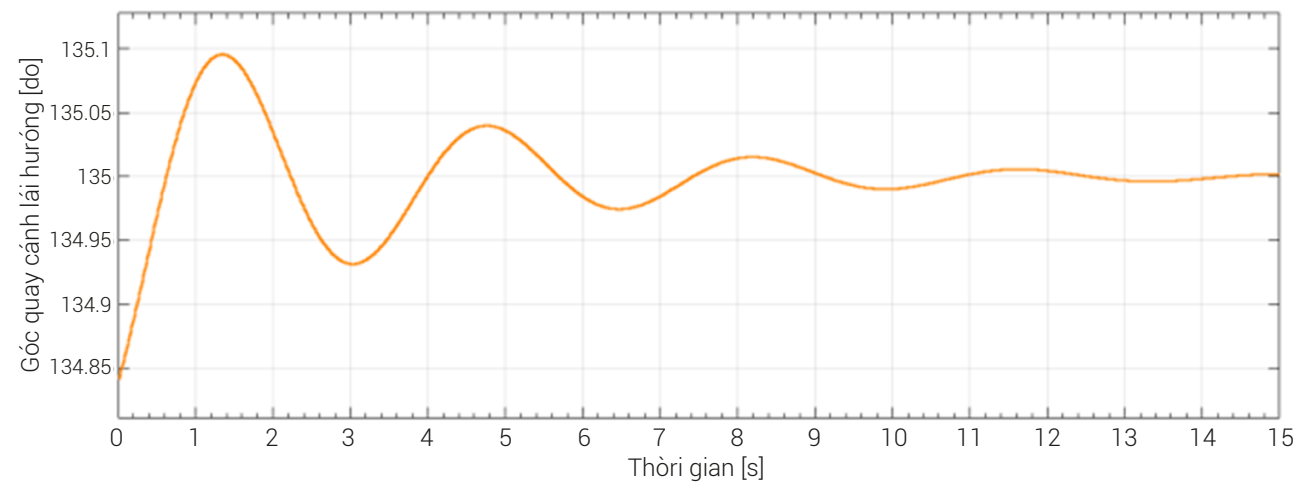

Figure 7. Rudder angle with PID control law (Kx reduction)

Source: own work 
Optimal LQG controller to adjust the rudder supplying water to the turbine of small and medium hydro power plants

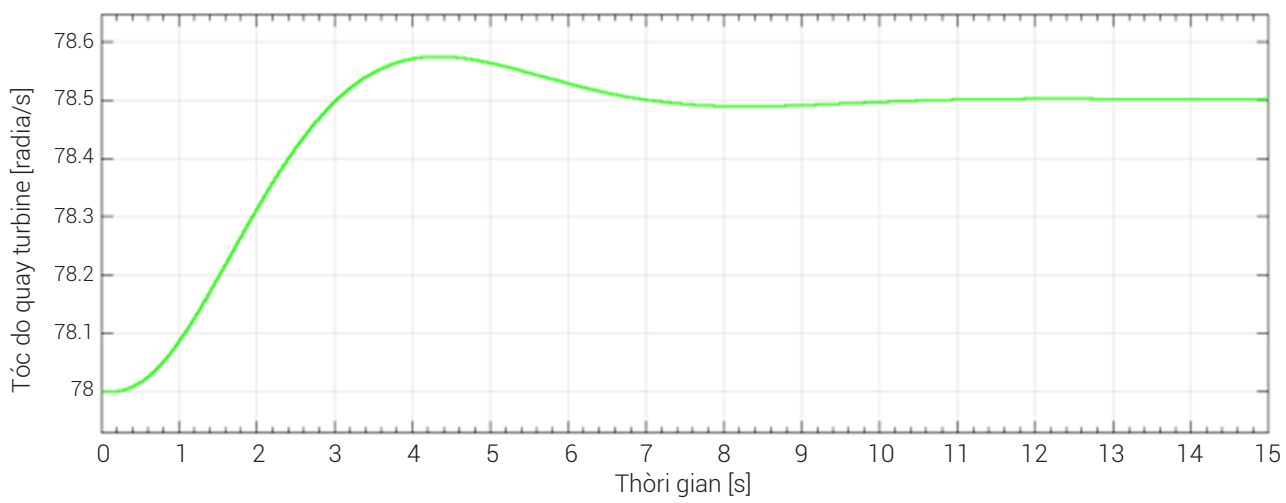

Figure 8. Turbine rotating frequency with PID control law (Kx increase) Source: own work

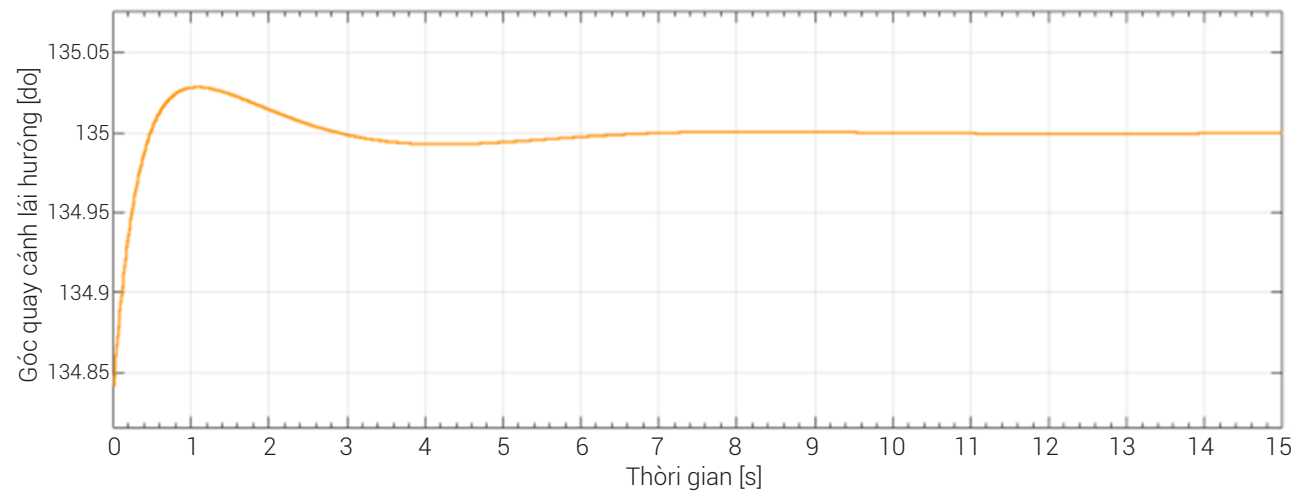

Figure 9. Rudder angle with PID control law (Kx increase) Source: own work

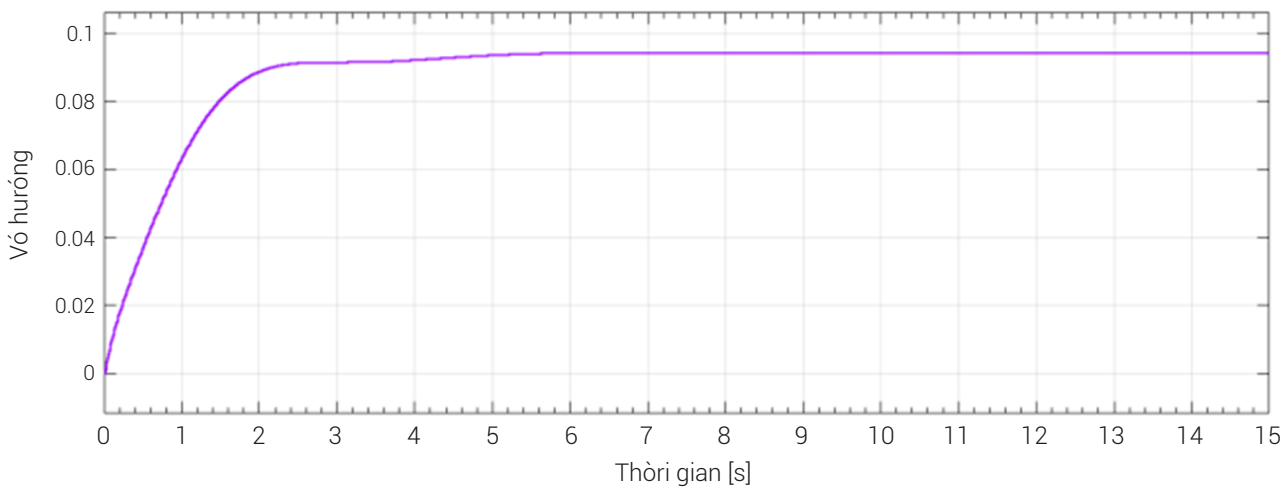

Figure 10. The objective function J with PID control law (Kx increase) Source: own work 
The above graphs show that, with optimal control: the turbine rotation frequency acheives the standard value with small fluctuations, the setting time is small, the objective function $\mathrm{J}$ is also small $(\mathrm{J}=0.0902)$. With PID control law, the objective function $\mathrm{J}$ also increases $(\mathrm{J}=0.11157, \mathrm{~J}=0,0947)$. This shows the superiority of the optimal control law.

\section{DISCUSSION AND CONCLUSIONS}

The use of renewable energies has been increasing in the recent years due to the current costs of oil and gas. Among them, hydroelectricity is the most developed renewable energy source throughout the world.

In $[13,14]$ a classical technique, known as Direct Power Control was used, but this method is not robust when presented with grid instability. Various research projects have been conducted in this field; for example [15] models a whole set of micro hydro power plants with electrical machines with control by fuzzy nonlinear systems. Although, the fuzzy technique guarantees stability, it should be noted that non-linear constraints lead to complicated calculations.

In this paper, by identifiying the kinematic model parameters and estimating the consumption load, we are able to set up the optimal control problem for the water supply flow direction into small and medium hydropower turbines. An LQG optimal control theory was applied, building an algorithm to determine the status coefficient matrix of the kinematic system and the load vector in the control command structure. The structure of the control command will determine the return request regarding hardware and software when designing and manufacturing the turbine - generator combination. The algorithm presented in the paper is the basis for setting up the software when designing and manufacturing the turbine - generator combination. Applying this algorithm, the process of adjusting the transmitted power according to the required load will be performed with quality.

\section{REFERENCES}

[1] Dang Tien Trung, Pham Tuan Thanh, "Control model for electric generators of medium and small hydroelectric factories," Journal of military science and technology, no. 50, pp.55-64, 2017. 
Optimal LQG controller to adjust the rudder supplying water to the turbine of small and medium hydro power plants

[2] D. Tiomo, R. Wamkeue, "Dynamic Modeling and Analysis of a Micro-Hydro Power Plant for Microgrid Applications," Electrical and Computer Engineering (CCECE) 2019 IEEE Canadian Conference, pp. 1-6, 2019.

[3] Dianwei Qian, Jianqiang Yi, Xiangjie Liu, Xin Li, "GA-based fuzzy sliding mode governor for hydro-turbine," Intelligent Control and Information Processing (ICICIP) 2010 International Conference, pp. 382-387, 2010.

[4] L.N. Giang, L. Kaipei, "Simple backstepping control design for the rotor-Side converter of a DFIG wind turbine generator," International Journal of Applied Mathematics and Statistics, vol. 51, no. 21, pp. 396-405, 2013.

[5] D. Q. Dang, S. Wu, Y. Wang, W. Cai, "Model predictive control for maximum power capture of variable speed wind turbines," Proceedings of the International Power Electronics Conference (IPEC '10), pp. 274-279, October, 2010.

[6] N.T.H. Yen, C. Hongkun, L.N. Giang, "Study on VSC-HVDC grid topology of offshore wind farms," Cluster Computing, 22, pp. 14803-14810, 2019.

[7] S. Nourdine, H. Camblong, I. Vechiu, G. Tapia, "Comparison of wind turbine LQG controllers using individual pitch control to alleviate fatigue loads," 201018 th Mediterranean Conference on Control \& Automation (MED), IEEE, 2010, pp. 1591-1596. doi:10.1109/MED.2010.5547822..

[8] Chalang Hamarasheed, Sallehuddin Mohamed Haris, Zulkifli Mohd Nopiah, "Weighted multiple model adaptive LQG and PI control for a hydro turbine plant," Advanced Mechatronic Systems 2012 International Conference, pp. 656-661, 2012.

[9] V.T. Hung, S.H. Chun, L.N. Giang, "The Control Method in Synchronous Frame for DVR to Mitigate the Balanced and Unbalanced Voltage Sag/Swells Phenomenon in Power Network," Advances in Intelligent Systems and Computing, 1129 AISC, pp.1-14, 2020.

[10] T.T. Ngoat, X.M. Zha, L.N. Giang, "Selecting optimal reactive compensation device for power system," Applied Mechanics and Materials, 556-562, pp. 1560-1563, 2014.

[11] Tran Huu Phuong, Mikhail P. Belov, Nguyen van Lanh, "State Estimation of Nonlinear Electromechanical System using Extended Kalman Filter”, IEEE Xplore, 2020.

[12] K. Szabat, T. Orlowska-Kowalska, K. Dyrcz, "Extended Kalman filters in the control structure of two-mass drive system," Bull. Pol. Acad. Sci. Tech. Sci., vol. 54, no. 3, pp. 315-325, 2006. 
[13] S. Breban, M. M. Radulescu, B. Robyns, "Direct Active and Reactive Power Control of VariableSpeed Doubly-Fed Induction Generator on Micro-Hydro Energy Conversion System," pp. 1-6, IEEE, 2010.

[14] C.P. Ion, C. Marinescu, "Control of parallel operating micro hydro power plants," Optimization of Electrical and Electronic Equipment, pp.1204-1209, 2010.

[15] Issam Salhi, Sald Doubabi, Najib Essounbouli, Abdelaziz Hamzaoui, "Application of multi-model control with fuzzy switching to a micro hydro-electrical power plant," Renewable Energy, Elsevier, Vol. 35, pp. 2071-2079, 2010. 